\title{
Rien de nouveau sous le soleil
}

\section{FLORENCE LOJACONO}

\author{
UNIVERSIDAD DE LAS PALMAS DE GRAN CANARIA \\ PÉREZ DEL TORO, 1 - 35003 LAS PALMAS DE GRAN CANARIA (ESPAÑA) \\ TÉL. +34928 4517 20; FAX +34928458050 \\ <flojacono@caramail.com>
}

RÉSUMÉ Touriste de bananes (1938) et Ceux de la soif (1938) occupent parmi les romans durs de Simenon une place à part. En effet le retour à la nature et l'échec qui le sanctionne en sont la trame commune. Le discours primitiviste instille son poison et la mort arrive avec ce constat : les rapports entre les hommes ne subissent aucun changement quand on les expose à différentes latitudes. L'exotisme n'existe pas.

MOTS-clÉs Simenon. Exotisme. Primitivisme.

\section{"Nada nuevo bajo el sol"}

RESUMEN Touriste de bananes (1938) y Ceux de la soif (1938) ocupan un lugar muy especial en las novelas duras de Simenon. El tema del retorno a la naturaleza y el fracaso que lo confirma es, en efecto, la trama común. El primitivismo destila su veneno y llega la muerte atestándose así que las relaciones entre los hombres no sufren ningún cambio cuando se les expone a diferentes latitudes. El exotismo no existe.

palabras clave Simenon. Exotismo. Primitivismo.

\section{"Nothing New Under the Sun"}

ABSTRACT Touriste de bananes (1938) and Ceux de la soif (1938) hold a special place among Simenon's 'hard' or 'serious' novels. Their common theme is the return to nature and its failure. Primitivism instills its poison and death comes as if to confirm that relationships between human beings don't change when we change latitude. Exoticism does not exist.

KEY WORDS Simenon. Exoticism. Primitivism. 


\section{INTRODUCTION}

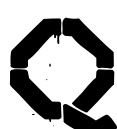

uelle est la couleur du polar ? Pour certains il tire vers le noir, pour d'autres, n'étant qu'une "forme abâtardie et foraine" (Boileau-Narcejac, $1975: 3$ ) du roman policier, la question ne mérite pas même d'être posée. Pourtant, une nouvelle couleur, le gris, se détache doucement de l'œuvre protéiforme de Georges Simenon (Baronian, 2002). Grise, la série des Maigret et gris les 117 romans que Simenon écrivit sans faire intervenir le célèbre commissaire et qu'il appelait les 'romans durs' ou les 'romans romans'. On ne peut séparer ces deux facettes de l'œuvre simenonienne. En effet, de nombreux points communs autorisent le rapprochement de la production strictement policière de la production romanesque. Le personnage du raté, souvent tourmenté par une inquiétude viscérale, et le motif de la fuite recouvrent l'ensemble des romans durs et des Maigret d'une teinte uniforme. Touriste de bananes (Simenon, 1938a) et Ceux de la soif' (Simenon, 1938b) sont des romans durs, plus précisément, ils appartiennent à la lignée des romans exotiques de Simenon. Le raté des villes y devient le "raté de l'aventure" (Simenon, 1938c), et le "mythe du bon clochard" (Richter, 1993: 82)y est relayé par celui du 'bon sauvage'. Ces deux récits proposent des mises en texte différentes d'un même thème, celui du retour à la nature, et d'un même constat : l'exotisme n'existe pas.

\section{LE RETOUR À LA NATURE}

Oscar Donadieu est venu chercher à Tahiti, dans Touriste de bananes, ce que les Allemands de Ceux de la soif croyaient encore possible aux Galapagos : une vie plus 'vraie' car plus proche de la nature. Ils sont tous,

1 L'histoire de ces Allemands établis aux Galapagos à la recherche d'une vie plus authentique est racontée dans Les Robinsons des Galapagos (Wittmer, 1960) récit autobiographique de Margret Wittmer (1904-2000) et fut reprise par Simenon sous deux formes différentes : dans une série d'articles pour Paris-Soir en 1935 et dans Ceux de la soif (1938). 
selon l'expression de Pierre Halen, des “chercheurs d'envers” (Halen, 1997 : 205) car ils ont fui la civilisation "en quête d'un mode alternatif de rapport à la Nature" (ibid.). L'Océanie a fourni à Simenon matière à deux autres romans, Long Cours (1936) et Le Passager clandestin (1947) mais le retour à la nature, bien que présent, n'est pas la raison essentielle du séjour des protagonistes aux antipodes : Mittel (Long Cours) et le major Owen (Le Passager clandestin) sont venus à Tahiti pour y rejoindre quelqu'un, à la différence d'Oscar Donadieu (Touriste de bananes) et des colons allemands (Ceux de la soif) qui eux, ont délibérément choisi leur île pour y vivre dans un dénuement voulu. Or, quand le dénuement allie profession de foi et mode de vie, on est devant un personnage emblématique : l'homme-nature. La figure de l'ermite malgré tout, se condamnant lui-même “à vivre de bananes et à s'habiller de toile à sac" (Chadourne, $1927: 768$ ) est déjà présente dans Vasco de Chadourne et elle réapparaît avec force dans La Tête coupable de Romain Gary (1968). L'idéal de l'homme-nature, disciple de la frugalité heureuse, est une vie en communion parfaite avec l'environnement naturel, le plus loin possible des hérauts de la consommation forcenée. C'est ainsi qu'Oscar Donadieu a choisi de s'établir en Polynésie parce qu'il “avait lu qu'à Tahiti on pouvait mener une vie naturelle, sans argent, sans contraintes, dans un décor idéal” (Simenon, 1938a : 378). De leur côté, Rita et le docteur Müller “étaient venus aux Galapagos pour se rapprocher de l'état de nature" (Simenon, 1938b :13). La condition insulaire des protagonistes de Ceux de la soif a encouragé un rapprochement de ce roman avec la robinsonnade (Racelle-Latin, 1980 : 60). En effet, à première vue, Robinson et l'homme-nature ont des modes de vie et des apparences similaires, mais seul l'homme-nature revendique son état comme une contestation ouverte, un reproche vivant adressé à la société de consommation. Entre Robinson et l'homme-nature, il y a plus qu'un naufrage de différence. Le naufragé traditionnel aborde l'île malgré lui, une infortune de mer lui imposant de survivre avec les moyens du bord; l'homme-nature quitte de son plein gré le naufrage de la société dans laquelle il vit, son credo lui imposant de ne vivre désormais qu'avec les moyens du bord. Mais pourquoi les candidats au dénuement volontaire sont-ils systématiquement tournés en dérision par ceux qui, à Tahiti ou ailleurs, les observent, d'un œil goguenard, débarquer avec plus d'illusions que de bagages? C'est la question que se pose Oscar peu après son arrivée : 
[...] pourquoi ce terme péjoratif touriste de bananes, appliquéà ceux qui, comme lui, rêvaient de se fondre dans la nature, de vivre en tête à tête avec elle et avec elle seule, en renonçant aux commodités de la civilisation ? (Simenon, 1938a : 290)

Son comportement d'homme-nature provoque parmi les Blancs de l'île des sentiments allant de la pitié à la moquerie, alors que lui "ne voulait pas de la pitié des autres, mais plutôt leur admiration" (id. :335.) Ce que ne sait pas Oscar, quand il pose son sac sur le quai du boulevard Pomaré, c'est que son histoire a déjà été écrite par tous ceux qui, partageant son idéal, l'ont précédé dans l'île. Et cette histoire, sans cesse réécrite, est celle d'un échec, car, quelles que soient les circonstances personnelles de ces nouveaux Saint-François, invariablement, après un mois ou au plus un an :

[...] on le verra arriver à l'office des passeports, les traits burinés, l'œil éteint, piteux, miteux, drapé dans un reste de dignité.

- On me rappelle en Europe, où j'ai un parent malade, dira-t-il pour s'excuser.

Ce n'est pas vrai. Il n'a même pas reçu de lettre. Mais il n'en peut plus. Il y a trop longtemps qu'il s'ennuie en tête à tête avec la nature, qu'il fait des efforts surhumains pour avaler les fades bananes et qu'il résiste à aller mendier un grand verre de bière au bistrot. (Simenon, $1938 \mathrm{c}: 60)$

Comme l'a fait Thor Heyerdahl. En effet, avant d'être le célèbre capitaine du Kon-Tiki, Heyerdahl a tenté aux Marquises l'expérience d'une vie en totale communion avec la nature. Mais il a dû se rendre à l'évidence : la vie naturelle est bien plus belle quand on la rêve devant la cheminée d'un chalet norvégien, que sous les piqûres des moustiques de Fatu-Hiva. La conclusion que tire Heyerdahl de sa tentative : "On ne peut acheter un billet pour le paradis” (Heyerdahl, 1974:355) pourrait très bien être la dernière ligne de Touriste de bananes ou de Ceux de la soif. Oscar, comme Heyerdahl a construit une île à la mesure de ses attentes : iln'a pu "s'empêcher de bâtir en rêve un décor qui ressemblait davantage au jardin du presbytère qu'à autre chose" (Simenon, 1938a : 378). La Polynésie et les Galapagos dans les romans exotiques de Simenon ont en commun d'être une projection des désirs des protagonistes, une construction mentale plus qu'un espace réel. La réalitélocale n'ayant que 
peu de rapport avec les attentes rêvées, on comprend pourquoi les touristes de bananes sont des "ratés de l'aventure, si vous voulez" (Simenon, 1938c : 15). Lors de ses voyages, Simenon a été frappé par l'égale répartition de ces "ratés de l'aventure" tout autour du globe, et principalement, tout au long des colonies françaises. Ce sont eux qui alimenteront ses "notes en marge d'un tour du monde" (id. : 9) rassemblées sous le titre La Mauvaise étoile (1938c). Le personnage du raté lui fournit aussi un titre : Touriste de bananes. Le touriste de bananes est la version tropicale du clochard de la métropole, les velléités rousseauistes en plus. Par delà le motif du retour à la nature, c'est donc le personnage du raté qu'illustrent Touristede bananes, Long Cours et Ceux de la soif. Oscar Donadieu veut se refaire une vie nouvelle à Tahiti, loin des lâchetés quotidiennes. Mais à défaut d'être fort comme Robinson, ou philosophe comme Rousseau, il ne sera qu'un touriste de bananes parmi d'autres. Un raté. Aux Galapagos aussi, la situation se dégrade rapidement. L'équilibre de l'île est rompu à l'arrivée d'une pseudocomtesse décidée à construire un havre de paix pour milliardaires surmenés : "l'Hôtel du retour à la nature" (Simenon, 1938b : 45). Que Simenon dans Ceux de la soif, adaptation romancée d'un fait réel, ait préféré rebaptiser le projet de la comtesse "Hôtel du retour à la nature" au lieu de reprendre l'appellation originale d'Hacienda Paradiso (Wittmer, $1960: 65)$ est révélateur de l'importance du thème. La comtesse est, elle aussi, une ratée de l'aventure : elle disparaîtra mystérieusement et son projet hôtelier ne verra jamais le jour. Oscar à Tahiti et les colons allemands aux Galapagos illustrent ce parcours tout en "dégringolade" (Simenon, 1938c : 80) caractéristique des milliers de "faibles, de malchanceux, de rêveurs, de lymphatiques ou de malades qui poursuivent une chimère en y croyant ou en n'y croyant plus” (id. : 13). $\mathrm{Au}$ bout de cette dégringolade : la mort. Oscar, ne pouvant plus supporter la solitude ni le mode de vie qu'il s'est lui-même imposé, préfère se suicider plutôt que d'entendre les rires gras saluer son échec, plutôt que de s'avouer n'avoir été, finalement, qu'un touriste de bananes parmi d'autres. 


\section{PRIMITIVISME ET EXOTISME}

Le désir d'Oscar et des colons allemands de revivre les premiers âges de l'humanité est né en métropole, à la lampe des bibliothèques. C'est un désir culturel, transmis par la littérature et la philosophie, et dont les origines remontent à la découverte du Nouveau Monde, ou plus exactement aux récits enthousiastes qui ont suivi les expéditions des grands navigateurs. Le retour à la nature est une émanation directe du primitivisme culturel européen tout autant que la preuve de la persistante efficacité de ses arguments. Quels sont les traits pertinents du primitivisme ? Todorov les énumère dans Nous et les autres : le dénuement, l'égalitarisme et la vie à l'écoute de la nature (Todorov, 1989: 304). Or n'est-ce pas exactement ce que recherchent Oscar et les colons allemands ? Le touriste de bananes est donc la variante primitiviste du "raté de l'aventure" (Simenon, 1938c:15). Que Simenon ait consacré un chapitre de ses notes aux "Touristes de bananes ou les Adams de Chicago et les Èves de Manchester et d'Oslo dans les nouveaux paradis terrestres" (id. : 57-67), que Romain Gary ait rebaptisé ironiquement les hommes-nature des "adams bananivores" (Gary, 1968 : 260) n'est pas fruit du hasard. Le primitivisme est en effet lié aux mythes des origines. Éric Vibart termine ainsi son essai sur Tahiti au siècle des Lumières :

Plus qu'une étude comparative entre sociétés sauvages et civilisées, le primitivisme culturel proposait en dernière analyse de résoudre les difficultés des sociétés occidentales en tenant compte de cette nature universelle et originelle de l'homme. (Vibart, 1987 : 225)

Derrière le thème du retour à la nature se lit en filigrane, celui de la nostalgie du paradis. Or la quête des premiers matins du monde et l'exotisme sont indissolublement associés dans l'imaginaire européen. Au XVI ${ }^{e}$ siècle, l'enchantement provoqué par les mers du Sud, s'il ne doit plus grand chose à la magie des Circé, n'en est pas moins tout aussi efficace. Les récits des découvreurs, en particulier depuis le Post-scriptum sur l'île de la Nouvelle Cythère publié en 1669 par Commerson, deux ans avant celui de Bougainville, ont remplacéles philtres anciens : désormais on lit la potion au lieu de la boire. C'est ainsi que, pour longtemps, 
primitivisme va rimer avec exotisme ${ }^{2}$. Touriste de bananes et Ceux de la soif, parce qu'ils exposent les illusions primitivistes au soleil des tropiques, parce qu'ils abordent l'exotisme par l'imagination désirante, sont sûrement les plus "primitivistes" des romans de Simenon. Il n'est pas étonnant que ces deux romans aient en commun le retour à la nature et l'exotisme : quel lieu aurait pu, mieux qu'une île du Pacifique, servir de décor au désir adamique des protagonistes ? En reformulant Lacassin on pourrait définir l'exotisme comme un peu de romantisme frivole greffé sur une motivation douloureuse (Lacassin, 1973:171). Quand les parfums sucrés s'évaporent, reste la douleur. Le soleil, s'il brûle la peau, s'il contribue à une éphémère autant qu'illusoire intégration du Blanc à la communauté insulaire, n'a aucun effet sur les petites lâchetés quotidiennes qui se révèlent aussi destructives à Paris qu'à Papeete. Oscar aurait voulu être un homme fort, ainsi que Mittel voulait être Mopps dans Long Cours, en fait il est "honteux de sa lâcheté" (Simenon, 1938a : 295) à Tahiti comme il l'était déjà en métropole. Il aurait voulu faire mentir les Cassandres de l'hôtel des Méridiens, mais il se rend à l'évidence "c'était comme ailleurs, comme dans n'importe quel pays" (id.: 349). Ce n'est même pas une révélation car,

Depuis le premier jour, pour tout dire, il avait compris que ce n'était pas possible, que la fameuse vie naturelle dont on lui avait parlé n'existait pas, que sa solitude n'était qu'une solitude de clochard, qu'il y avait ici comme partout, des règles à suivre et qu'il ne faisait, en somme, avec tout son héroïsme, que jouer au boy scout à quelques pas du village. (id.: 365)

Pour Pierre Halen, la singularité de Simenon par rapport à l'écriture des colonies est qu'"il a vu qu'il pouvait parfaitement déplacer outre-mer les milieux sociaux qu'il représente le plus souvent en métropole" (Halen, 1997 : 207). En élargissant le concept, il est possible de remplacer l'outre-mer des colonies françaises par tout lieu perçu

2 Les spécialistes s'accordent généralement à faire remonter l'association du primitivisme et de l'exotisme à la lettre écrite par Amerigo Vespucci à Lorenzo di Pier Francesco dei Medici, lettre qualifiée par Todorov de best-seller de la littérature de voyage au XVI siècle (Todorov, 1989:300). Traduite en latin sous le nom de Mundus Novus, cette lettre connut une large diffusion aux premières heures de la Renaissance. 
comme exotique. Ainsi le drame humain qui se déroule à Tahiti dans Touriste de bananes ne diffère en rien de celui qui se joue au sein de la petite communauté de Ceux de la soif. Changer de latitude n'est pas changer qui on est. Et c'est de ce constat que meurt Oscar. Si la solitude est pour l'individu isolé un vain recours à son mal être, elle agit comme del'amadou sur les groupes sociaux, enflammant les moindres tensions. Aux Galapagos, une poignée d'individus a suffi à recréer tout l'édifice de la hiérarchie sociale, flatterie rancœur et mépris compris. C'est ainsi que sur Floréana Herrmann parle toujours avec déférence à Müller, exactement comme s'ils étaient dans un salon de Berlin. La raison "venait d'Allemagne avec eux" (Simenon, 1938b:10). En effet, avant de s'installer aux Galapagos, Herrmann n'était que simple préparateur à l'université de Bonn, alors que Müller était un éminent médecin de Berlin. Comme Oscar, Herrmann porte en lui, où qu'il aille, la raison de son inquiétude. Quand arrive la comtesse von Kleber, elle s'auto proclame immédiatement "souveraine de Floréana" (id.: 18) puis "impératrice des Galapagos" (id.:155). En accord avec son titre et ses ambitions, elle traite les membres de sa suite et les habitants de l'île en sujets. Le jeune Kraus passe du statut de sigisbée à celui de "domestique" (id. : 46, 71, 117, 152, 155) quand à Müller, il se demande si les Herrmann allaient "devenir, comme Kraus, les serviteurs de la comtesse ?” (id. : 51). Le dépaysement ne rend que plus patent l'impossibilité de se changer soi-même, l'impossibilité de recommencer ailleurs une vie gauchie dès l'origine. L'échec d'Oscar et celui de la comtesse signent la victoire du gris. La beauté du paysage, bien que réelle, et à laquelle d'ailleurs l'écrivain est sensible, n'est pas une condition suffisante au bonheur car "on ne peut à tout moment avoir conscience qu'on vit dans le plus beau paysage du monde" (Simenon, 1938a:335). Bientôt les journaux de la métropole vous attirent plus que les couchers de soleil sur le lagon. L'imagination désirante des exilés volontaires s'était contentée de la clémence du climat et de la supposée bienveillance de la nature pour transformer un îlot tropical en un écrin étincelant $t^{3}$. Mais voilà, "le Pacifique, à cet endroit, est gris, lui aussi ; le ciel est gris. C'est une erreur de croire à la gaîté, à la

3 Voir parexemple les nombreux adjectifs utilisés pour décrire les paysages de Tahiti. Voir aussi les lignes que Bernard Alavoine consacre à la lumière et aux couleurs dans son étude de l'œuvre de Simenon (Alavoine, 1998: 58-66). 
lumière des tropiques" (Simenon, 1938c:33). La grisaille parisienne, celle de Berlin, celle que l'on emmène partout avec nous et qui est faite de nos inquiétudes et de nos rancœurs, suffit à rendre opaques les rivages les plus lumineux. Les îles tropicales ont, chez Simenon, le goût de l'amertume et lui dictent ce constat : "l'exotisme n'existe pas" (id.: 33). Le même jugement, dans une version plus ironique, est porté trente ans plus tard par Gary dans La Tête coupable. On retrouve dans ce roman de nombreux échos simenoniens en plus du personnage de l'hommenature : la même attraction exercée par les journaux sur les exilés volontaires ou non et le personnage du contemplatif. Dans La Mauvaise étoile, le vicomte de Simenon vit dans une église de Nouvelle-Calédonie et reste des heures assis à regarder la mer, dans La Tête coupable, le Baron est, à Tahiti, est une sorte de dieu immobile, face à la mer. Simenon et Gary, des anti-Loti ${ }^{4}$ par excellence, ramènent l'exotisme à sa plus stricte expression : un paysage réduit à ses couleurs et à ses parfums, des hommes dont les poses oscillent entre la supercherie et la détresse.

\section{UN ANTIDOTE MORTEL}

Le thème du retour à la nature et la quête d'un "idéal beau et propre" (Simenon, 1938a :289) motivent dans Touriste de bananes et Ceux de la soif un comportement récurrent dans les romans de Simenon : la fuite. Oscar "voulait fuir les hommes et vivre une vie simple" (id. : 299), quant au docteur Müller, encore à Berlin, il annonce un jour : “je pars pour vivre le reste de mes jours dans une île déserte du Pacifique..”.(Simenon, 1938b : 29). Alors que certains 'romans durs', comme Le Voyageur de la Toussaint (Simenon, 1941), se terminent de manière optimiste sur une fuite librement choisie ouvrant vers des horizons réellement plus accueillants, celle des "ratés de l'aventure" (Simenon, 1938c : 15) est sanctionnée par le drame. La fuite est un mouvement né du désir des protagonistes, elle trace sur le globe la trajectoire de leurs attentes. L'exotisme et la fuite sont deux formes du détachement du soi, ou plutôt deux tentatives de réponse à l'inquiétude

4 En parlant des romans exotiques de Simenon, Maurice Dubourg souligne "ce côté antiLoti d'une œuvre souvent marquée de pessimisme” (Dubourg, 1973 : 155). 
qui étreint les protagonistes de Simenon, et pas seulement ceux des romans dit exotiques puisque, selon Dumortier, "tous les héros de Simenon, excepté Maigret ... sont des solitaires et des angoissés" (Dumortier, 1985:8). Le rôle joué par la fuite dans ces deux fictions est à souligner. En effet, la fuite des personnages de Simenon n'est pas simple force centrifuge, elle relève bien plus d'une inquiétude existentielle que de circonstances anecdotiques. Francis Lacassin place d'ailleurs Touriste de bananes et Ceux de la soif au nombre des romans illustrant le thème de la fugue initiatique (Lacassin, 1973 : 157-183), motif qui, selon lui, "hante l'œuvre de Simenon depuis plus de trente-cinq ans” (id. : 158). Oscar Donadieu n'en est pas à sa première fugue (Simenon, 1938a :377), il avait "déjà tenté de fuir" de nombreuses fois (id.: 289) avant que son dernier voyage ne le conduise à Tahiti. Le retour à la nature et les exigences d'une pauvreté heureuse imposent aux protagonistes l'abandon volontaire et définitif du lieu d'origine, désormais ressenti comme incompatible avec leur nouvelidéal. La fuite, nous l'avons dit, est cependant plus qu'une simple force centrifuge aux ordres d'un exotisme de pacotille. Oscar lui-même s'en rend compte : “Ce n'est pas moi qui fuis! Je vais vers quelque chose" (id. : 303). Avec JeanMarc Moura (1998), le lien est fait entre la fuite et l'exotisme. En effet, le concept de fuite fait le départ entre l'exotisme nostalgique, celui de L'Invitation au voyage et l'exotisme impérial, celui de Robinson Crusoé. Dans la littérature de l'exotisme nostalgique c'est l'ici-bas qui est un exil -la France pour Oscar, l'Allemagne pour les Herrmann, Muller et Ritaalors que l'ailleurs -Tahiti dans Touriste de bananes, les Galapagos dans Ceux de la soif- est paré des ornements de la vie authentique. La fuite, telle qu'elle est vécue par les protagonistes, nous ramène ainsi à la nostalgie des origines, déjà évoquée à propos du retour à la nature. L'île lointaine devient la promesse du lieu idéal, originel, où rien n'est encore corrompu, où tout est encore possible, un "sur-lieu" selon l'expression de Moura (Moura, 1998 : 267). Or on ne peut vivre dans un "sur-lieu”, un tel lieu n'existe que dans l'imagination désirante. Tout ce que ce lieu a, en plus, de beauté, il l'a, en moins, de réalité. Sa beauté d'ailleurs ne se confond qu'à première vue avec les couleurs chatoyantes et les parfums fleuris, sa réelle et seule beauté est d'être une promesse : la promesse de pouvoir recommencer. En fuyant, les protagonistes tentent de renverser la vapeur dans cette machine infernale qu'est leur propre destinée. Plusieurs indices, dans ces deux romans, nous font sentir le poids du 
destin : de la nostalgie des origines travestie en retour à la nature à la fugue initiatique qui est un défi envers soi comme envers la société. $\mathrm{Si}$, comme le dit Moura, "les figures nostalgiques sont des chrysalides du destin" (id. : 349) on comprend mieux ce que voulait dire Oscar quand il affirmait ne pas fuir mais aller vers quelque chose. Oscar-chrysalide cherchait la terre sans mal où il aurait pu renaître, quitter sa vieille enveloppe de faible et de lâche. Le destin est omniprésent dans les romans de Simenon, il est même "l'élément perturbateur dans le schéma narratif des romans durs” (Alavoine, 1998: 93). Il n'est donc pas étonnant que le guide des romans et nouvelles de Simenon (Piron, 1983) renomme “les 117 romans durs : romans de la destinée" (Piron, 1983 : 17). C'est par cette tension, par le côté existentiel des drames simenoniens que les "romans durs" s'apparentent au genre policier. En effet, le "roman dur" est bien une catégorie du polar car "il présente lui aussi la matière d'une enquête, qu'il revient au lecteur de mener à bien" (Dumortier, 1985: 10) C'est ainsi que dans les "romans durs" la charge de l'enquête revient au lecteur. Mais c'est d'une enquête toute particulière qu'il s'agit. Pas de crime dans Touriste de bananes mais un suicide ; un crime supposé mais pas de cadavres dans Ceux de la soif. Pas de crime non plus dans Long Cours ni dans Le Passager clandestin, les deux autres romans tahitiens de Simenon. Quelle est alors cette enquête qu'il revient au lecteur de mener à bien ? Il s'agit de remonter de l'échec final des divers protagonistes à une commune inquiétude existentielle, de la multiplicité anecdotiques des faits à l'unicité essentielle de la cause. L'inquiétude qui fait d'eux des exilés ici-bas et leur enjoint de fuir a pourtant son antidote : le retour au temps des origines, ce temps que Mircea Eliade définit comme "le Temps primordial, le Temps où l'événement a eu lieu pour la première fois” (Éliade, 2002 : 33). Le Grand Temps s'oppose au temps profane, chronologique et quotidien. Il est le temps de la perfection des commencements, celui qu'Oscar et les Allemands pensent trouver sur l'île. Le fait que les romans les plus 'primitivistes' de Simenon, donc ceux qui, le plus explicitement ont recours à la nostalgie des origines, soient des fictions insulaires n'est pas sans intérêt. L'île paradisiaque est, en effet, éloignée à la fois dans le temps, puisque qu'elle remonte au Grand Temps d'avant la faute, et dans l'espace, puisque sa localisation est incertaine, oubliée, et, dans tous les cas, lointaine. Or de ces deux éloignements, que l'on peut sans peine nommer deux exotismes, l'éloignement géographique, comme le dit Segalen, est le plus grossier 
(Segalen, 1978 : 96). Seul l'éloignement dans le temps est garant de l'authentique dépaysement que recherchent Oscar et les colons allemands. C'est à ce moment de l'enquête que le lecteur doit se faire fin limier. Puisque c'est l'échec qui attend les protagonistes, quelle a étéleur erreur? Quand cet échec se solde par la mort, qui a été l'instigateur du 'crime' ? La réponse est à chercher dans le rapport entretenu par les personnages avec le temps. Le Grand Temps, s'il est le temps des origines, est aussi celui du présent absolu. Or le présent absolu est la négation des changements inhérents au temps qui passe.C'est pourquoi il s'agit d'abord pour les protagonistes d'abolir le temps. Une façon triviale d'abolir le temps est tout simplement d'enlever sa montre (Lojacono, 2006:228): les colons allemands n'ont pas de montre et quant à Oscar "il y avait longtemps que sa montre ne marchait plus" (Simenon1938a : 351). Mais il est simpliste de croire abolir le temps en enlevant sa montre, comme il est simpliste de croire qu'ailleurs sera différent d'ici. La montre est au Grand Temps ce que les palmiers sont à l'Exotisme : un indice trivial, mis ostensiblement sur le chemin des protagonistes afin de les mettre l'épreuve. Les écrits de Simenon disent haut et clair que l'exotisme géographique n'existe pas, ses personnages prouvent que l'exotisme nostalgique est voué à l'échec. Le refus du confort moderne n'a assuré le bonheur ni aux Allemands, ni à Oscar. Leur faute a été de confondre retour au Grand Temps et retour dans le temps, de vouloir placer l'immanence des origines sur une frise chronologique. Retrouver la plénitude du Grand Temps n'a rien à voir avec le retour à la nature. Pensons à la flèche de Zénon et imaginons que le Grand Temps soit le mouvement de la flèche. On peut alors penser diviser le Temps qui passe en un grand nombre de moments, de moments si courts qu'il serait possible de chacun les confondre avec le présent. Retrouver le Grand Temps est alors vivre chaque instant avec une conscience accrue comme l'apprennent les palanais dans le dernier roman d'Huxley, Island (1962). Remarquons que cette façon d'aborder le temps comme une succession de plénitudes n'est pas sans rappeler l'existentialisme de Kierkegaard. Mais ce n'est pas la voie suivie par les protagonistes. Pour eux, la division mythique entre temps profane et temps sacré s'est transformée en division entre le temps du quotidien (celui d'avant la fuite) et le temps exotique (celui d'après la fuite). Première erreur puisque l'exotisme n'existe pas. Ensuite, loin de retrouver le Grand Temps des origines, ils fondent sous les tropiques un 
autre quotidien, qu'ils pensent d'autant plus 'vrai' que plus éloigné du confort moderne. Seconde erreur puisque le Grand Temps n'est pas le 'bon vieux temps' mais une conscience accrue du présent. S'enfuir dans les mers du Sud, enlever sa montre, sont de vaines tentatives de renouer avec une vie que l'on sent de plus en plus étrangère. C'est pour cela qu'avec Simenon nous pouvons dire qu'il n'y rien de plus ressemblant à un bar de Paris qu'un bar de Nouvelle-Calédonie. L'internationale des "ratés du Café du commerce" (Simenon, 1938c :14) prouve que, non seulement l'exotisme n'existe pas, mais qu'il est dangereux. C'est un antidote frauduleux, un poison subtil au goût de frangipanier. S'ily a eu 'crime', si Oscar se suicide, si la pseudo comtesse disparaît mystérieusement, c'est qu'ils ont tous bu de ce breuvage sucré dans le but d'apaiser leurs angoisses. Et c'est le primitivisme qui le leur a mis entre les mains.

\section{CONCLUSION}

Sous l'appellation péjorative de “touriste de bananes" et, plus généralement, derrière la tentation primitiviste des protagonistes des romans exotiques, affleurent toujours, et quelque soit la gaieté des décors mis à disposition, l'échec, la solitude, la mort, en un mot : le gris. Le thème du retour à la nature exposé dans les deux romans primitivistes de Simenon, Touriste de bananes et Ceux de la soif, est à la fois l'indice d'une inquiétude existentielle et la tentative d'y remédier. Le concept de retour à la nature entendu comme recul dans le temps et perçu comme une vie plus vraie contient en lui-même le germe de son échec dans la confusion associant bonheur et vie champêtre. Si l'exotisme n'existe pas c'est parce qu'il est inutile : les tropiques jamais n'allègeront les protagonistes du poids de leur destinée. Les personnages des romans insulaires, Mittel (Long cours), le major Owen (Le Passager clandestin), Oscar (Touriste de bananes), Herrmann (Ceux de la soif) invitent le lecteur à une réflexion sur les rapports entre ce que l'on est et ce que l'on aurait voulu être. Tous veulent recommencer ailleurs une vie dont ils seraient entièrement les maîtres, aucun ne réussira. Sous le soleil de l'Océanie le gris simenonien devient éclatant. Le seul effet produit par l'exotisme communément compris est de servir de contraste, par sa lumière et ses couleurs, à la grisaille quotidienne, elle aussi communément comprise 
FLORENCE LOJACONO

RIEN DE NOUVEAU

SOUS LE SOLEIL

comme étant la vie que menaient les protagonistes avant leur fuite. La mort, le suicide ou la disparition interviennent quand justement ceux-ci se rendent compte que le gris qu'ils pensaient fuir n'était pas autour d'eux, à Paris où à Berlin, mais en eux. 


\section{RÉFÉRENCES BIBLIOGRAPHIQUES}

ALAVOINE, Bernard (1998) Georges Simenon. Parcours d'une œuvre, Amiens, Encrage. BARONIAN, Jean-Baptiste (2002) Simenon ou le roman gris, Paris, Textuel.

BOILEAU, Pierre, NARCEJAC, Thomas, (1975) Le Roman policier, Paris, PUF, (1992).

CHARDOURNE, Marc (1927) "Vasco", Polynésie. Les archipels du rêve, Paris, Omnibus, pp. 703-835, (1996).

DUBOURG, Maurice (1973) “Géographie de Simenon”, Simenon, Paris, Plon, pp. 139-183.

DUMORTIER, Jean-Louis (1985) Georges Simenon, Bruxelles, Éditions Labor.

ELIADE, Mircea (1963) Aspects du mythe, Paris, Gallimard (2002).

GARY, Romain (1968) La Tête coupable, Paris, Gallimard (2000).

HALEN, Pierre (1997) "Proposition sur l'exotisme, avec une esquisse de Simenon en écrivain colonial', Traces 9, Georges Simenon et l'exotisme, Liège, Centre d'Études Georges Simenon, pp. 193-208.

HEYERDAHL, Thor (1974) Fatu Hiva. Le retour à la nature, Paris, Éditions du Pacifique (1976).

HUXLEY, Aldous (1962) Island, New York, HarperCollins (2002).

LACASSIN, Francis, SIGAUX, Gilbert (1973) Simenon, Paris, Plon.

LOJACONO, Florence (2006) Le Roman de l'île, Thèse de Doctorat, Université de HauteAlsace, Mulhouse.

RACELLE-LATIN, Danièle (1980) "De Pedigree aux romans psychologiques. Approche d'une signifiance”, Lire Simenon. Réalité/Fiction/Écriture, Bruxelles, Éditions Labor, pp. 51-78.

SIMENON, Georges (1936) Long cours, Paris, Gallimard (1966).

SIMENON, Georges (1938a)"Touristes de bananes ou Les Dimanches de Tahiti", Tout Simenon 21, Paris, Presses de la Cité, pp. 273-380 (2002).

SIMENON, Georges (1938b) Ceux de la soif, Paris, Gallimard (2001).

SIMENON, Georges (1938c) La Mauvaise étoile, Paris, Gallimard (2003).

SIMENON, Georges (1947) Le Passager clandestin, Paris, LGF (2001).

SIMENON, Georges (1977) Le Voyageur de la Toussaint, Paris, Gallimard (1941).

PIRON, Maurice (1983) L'Univers de Simenon : guide des romans et nouvelles (1931-1972) de Georges Simenon, Paris, Presse de la Cité.

VIBART, Éric (1987) Tahiti. Naissance d’un paradis au siècle des Lumières, Bruxelles, Éditions Complexe.

WITTMER, Margret (1960) Les Robinsons des Galapagos, [Postlagernd Floreana. Ein außergewöhnliches Frauenleben am Ende der Welt, 1960], Paris, Albin Michel. 
